\title{
Comparison of Short-term efficacy of valproate sustained- release between adult and children as first-line monotherapy in management of partial epilepsy: A multicenter observational open-label study
}

\author{
Afzal Momin', Ahsanul Habib², AKM Akramul Haque Tarik², Mohiuddin A Sikder, \\ Shaheen Akter ${ }^{5}$ \\ ${ }^{1}$ Assistant Professor, Department of Clinical Neurology, National Institute of Neurosciences \& Hospital, Dhaka, Bangladesh; \\ ${ }^{2}$ Professor, Department of Psychiatry, Anwar Khan Modern Medical College \& Hospital, Dhaka, Bangladesh; \\ ${ }^{3}$ Assistant Professor, Department of Psychiatry, Dinajpur Medical College \& Hospital, \\ Dinajpur, Bangladesh; ${ }^{4}$ Assistant Professor, Department of Psychiatry, \\ Chittagong Medical College \& Hospital, Chittagong, Bangladesh; \\ ${ }^{5}$ Professor, Department of Paediatric Neurology, \\ Bangabandhu Sheikh Mujib Medical \\ University, Dhaka, Bangladesh
}

(Received: July 2014; Revised: September 2014; Accepted: 1 January 2015)

\begin{abstract}
Background: Management of partial epilepsy is an important issue in the field of neurology. Objective: The purpose of the present study was to collect additional clinical data on efficacy and safety of sustained release sodium valproate chrono formulation as first-line monotherapy in patients newly or recently diagnosed with partial epilepsy in Bangladesh under daily practice condition. Methodology: This open-label, multicenter, non-controlled, prospective, observational study enrolled adults and children ? 6 years newly diagnosed with partial epilepsy with or without secondary generalization between March 2010 and February 2011. Patients were treated with sustained release sodium valproate. Primary evaluation criterion was the remission rate i.e. proportion of seizure-free patients at 6 months. Secondary evaluation criteria included retention rate at 6 months, remission rate at 1 and 3 months, investigator's global clinical impression rating, safety profile assessment. Results: A total of 185 adults and 115 children with mean 4.4 months duration of epilepsy were included. At inclusion the mean daily valproate dose in children was $329 \mathrm{mg}$ and $568 \mathrm{mg}$ in adults. The mean treatment duration in both children and adults was 5.5 months. At the end of 6 months $74.7 \%$ of the patients were seizure-free (children and adults; $79.1 \%$ vs $71.9 \%)$. The number of seizure free patients at 1 month was $109(36.3 \%)$ and $175(58.3 \%)$ at month 3 . The treatment retention rate at 6 months was $87.3 \%$ with small higher trend in adults than children ( $88.6 \%$ vs. $85.2 \%)$. Among the patients $9.3 \%$ experienced side effects like drowsiness and weight gain. No severe adverse event was reported. Conclusion: Sodium valproate sustained release formulation is effective for the first-line treatment of partial epilepsy in both adults and children with acceptable tolerability. [J Natl Inst Neurosci Bangladesh 2015;1(1): 2-4]
\end{abstract}

Keywords: Valproate; monotherapy; epilepsy

Correspondence: Dr. Afzal Momin, Assistant Professor, Department of Clinical Neurology, National Institute of Neurosciences \& Hospital, Sher-EBangla Nagar, Dhaka-1207, Bangladesh; Email: afzal4474m@yahoo.co.in; Cell no.: +8801716802081

Conflict of interest: Sanofi-Aventis Bangladesh Ltd. has given the financial support to the researchers to conduct the work.

Funding agency: The study was supported by a Sanofi-Aventis Bangladesh limited.

Contribution to authors: AM, AH, AKMAHT, MAS \& SA were contributed from protocol preparation to data collection. AM has prepared the manuscript and revised by SA.

How to cite this article: Momin A, Habib A, Tarik AKMAH, Sikder MA, Akter S. Comparison of Short-term efficacy of valproate sustained-release between adult and children as first-line monotherapy in management of partial epilepsy: A multicenter observational open-label study, J Natl Inst Neurosci Bangladesh 2015;1(1): 2-4

\section{Introduction}

Epilepsy is a disorder of the brain that is characterized by an enduring predisposition to generate seizures and by its neurobiological, cognitive, psychological, and social consequences $^{1}$. Around 30 to $40 \%$ of patients have seizures that are generalized at onset and rest is partial seizures. Sodium valproate has been widely used in treatment of epilepsy for nearly 40 years due to its wide spectrum of efficacy and relatively good tolerance ${ }^{2}$. For the treatment of generalized epileptic syndromes sodium valproate is recognized as the drug of first choice ${ }^{3-5}$. On the other hand, in focal onset or partial epilepsy syndromes, carbamazepine is the most widely used agent in first-line treatment ${ }^{6}$. The effectiveness of valproate in controlling partial seizures has been shown comparable to that of carbamazepine in a number of randomized, controlled clinical trials ${ }^{7-10}$. 
Sustained release formulation of sodium valproate has also been shown effective as first-line monotherapy in focal onset epilepsy with acceptable tolerability ${ }^{11}$. Epilepsy is a common neurological condition in Bangladesh. Though there are no national statistics, it is estimated that approximately 1.5 to 2.0 million people are suffering from epilepsy. For treating partial epilepsy carbamazepine is also conventionally used as first-line treatment in neurological practice. The objective of this observational study to collect additional clinical data on efficacy and safety of sustained release sodium valproate chrono formulation as first-line monotherapy in patients newly or recently diagnosed with partial epilepsy in Bangladesh under daily practice condition.

\section{Methodology}

This open-label, multicenter, non-controlled, prospective, observational study was conducted in a daily neurological practice condition in Bangladesh. In this study 300 patients with partial epilepsy who met inclusion criteria were consecutively enrolled by 5 investigators between March 2010 and February 2011. Adults and children 6 years and above, newly or recently diagnosed with partial epilepsy, with or without secondary generalized seizures for whom physicians decided to prescribe valproate sustained-release as first-line monotherapy were included in this study on receiving consent from patients or guardians for children. Newly diagnosed case was defined as at least 2 and no more than 10 partial seizures with or without secondary generalization during the past 6 months. Patients with primary generalized seizures or generalized epilepsy syndromes (Juvenile Myoclonlic Epilepsy), history of nonepileptic seizures were excluded to participate in this study. Exclusion criteria were also included pregnancy, lactation, hypersensitivity to valproate, liver disease, active CNS illness also those taking valproate 30 days prior to enrolment in this study. The patients were prescribed sustained release formulation of sodium valproate (Epilim Chrono $($ ) according to local prescribing information and on investigator's discretion. Dose adjustment was also made at investigator's decision to achieve maximal therapeutic benefit (free from seizure) for individual patient. Treatment duration was for 6 months. Patients were followed-up at 1, 3 and 6 months. Patient data was collected by the investigators at baseline and during follow-up visits using paper copy of Data Collection Forms (DCF) provided for each patient. After completion of the study (i.e. after 6 months) the patient was managed according to the decision of the physician. The primary endpoint evaluation criterion was the remission rate defined as the proportion of seizurefree patients at 6 months. Secondary endpoints were the proportion of seizure-free patients at 1 and 3 months, the retention rate defined as the proportion of patients continuing treatment at 6 months, neurological status described from brief neurological evaluation, Clinical Global Impression. Subgroup efficacy outcome were also evaluated according to age group (children $<15$ years or adults), seizure type (simple partial, complex partial or secondary generalized tonic-clonic seizures), and aetiology (idiopathic, symptomatic or cryptogenic). Patient's overall assessment to the therapy was evaluated at the end of the study. Safety was assessed by recording of spontaneouslyreported adverse events throughout the study. Adverse events were classified according to severity, and relationship with treatment. Statistical analysis was based on intention to treat (ITT) population who were included in the study and exposed to at least one dose of treatment. Statistical analysis was mainly descriptive and was summarized as mean, median, standard deviation, minimum, maximum and percentages. The efficacy outcome measures were estimated at $95 \%$ confidence interval and compared with chi-square test. Statistical analysis was done using SPSS 17.0.

\section{Results}

All of the enrolled 300 patients took at least one dose of valproate therapy and were considered as ITT population. Among the patients 38 (12.7\%) discontinued the study and the reasons were lost to follow-up (7.7\%), lack of drug efficacy $(2.3 \%)$ and poor compliance to protocol $(2.7 \%)$.

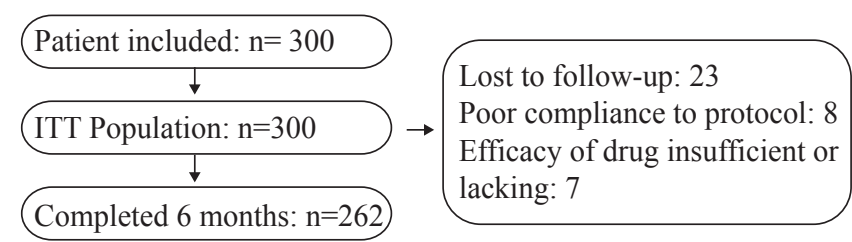

Figure1: Patient flow chart

One hundred and fifteen $(38.3 \%)$ of the ITT population were children (15 years) and $185(61.7 \%)$ were adults with higher male representation $(71.3 \% \& 83.7 \%)$. Of the patients 46 $(15.3 \%)$ had relevant medical history of encephalitis, head injury, febrile illness, birth trauma or premature birth though no abnormality was reported by the physicians on brief neurological examination. At inclusion the mean duration of epilepsy was 4.4 months (SD 0.8 months) at inclusion. The most common seizures type reported was complex partial seizures $(46 \%)$ and etiologically seizures were frequently idiopathic $(41 \%)$ in nature. The topographic localization was not determined by the investigators. The demographic and clinical characteristics of study subjects are presented in Table 1.

Table 1: Baseline characteristics of subjects at inclusion

\begin{tabular}{|c|c|c|}
\hline Baseline characteristics & $\begin{array}{l}\text { Age }<15 \\
(\mathrm{n}=115)\end{array}$ & $\begin{array}{l}\text { Age }>15 \\
(\mathrm{n}=185)\end{array}$ \\
\hline \multicolumn{3}{|l|}{ Age (years) } \\
\hline Mean $\pm \mathrm{SD}$ & $10.2 \pm 2.6$ & $30.8 \pm 12.2$ \\
\hline Median & 10.1 & 28.3 \\
\hline \multicolumn{3}{|l|}{ Gender } \\
\hline Male/Female & $82 / 33$ & $155 / 30$ \\
\hline \multicolumn{3}{|l|}{ Weight (kg) } \\
\hline $\mathrm{M} \quad$ ean $\pm \mathrm{SD}$ & $23.2 \pm 7.1$ & $53.03 \pm 12.83$ \\
\hline \multicolumn{3}{|l|}{ Diagnosis of epilepsy (months) } \\
\hline Mean \pm SD & $4.4 \pm 0.8$ & $4.5 \pm 0.9$ \\
\hline Median & 4.4 & 4.4 \\
\hline \multicolumn{3}{|l|}{ Type of seizure } \\
\hline Simple Partial & $39(37.1 \%)$ & $66(62.9 \%)$ \\
\hline Complex Partial & $58(42.0 \%)$ & $80(58 \%)$ \\
\hline $\begin{array}{l}\text { Secondary generalized } \\
\text { tonic clonic }\end{array}$ & $31(27.2 \%)$ & $83(72.8 \%)$ \\
\hline \multicolumn{3}{|l|}{ Etiology } \\
\hline Idiopathic & $54(18 \%)$ & $69(23.0 \%)$ \\
\hline Not determined & $61(20.3 \%)$ & $116(38.7 \%)$ \\
\hline
\end{tabular}


The mean daily valproate dose prescribed in children at inclusion was $328.7 \mathrm{mg}$ (SD $129 \mathrm{mg}$; median $300 \mathrm{mg}$; range $200-800 \mathrm{mg}$ ) and $568 \mathrm{mg}$ (SD $209 \mathrm{mg}$; median $500 \mathrm{mg}$; range $300-1000 \mathrm{mg}$ ) in adults. The mean treatment duration in both children and adults was 5.5 months (SD 1.2 months; median 6 months; minimum 1.4 to maximum 6 months). At 6 months of therapy the overall remission rate was $74.7 \%(224)$. In children the rate was significantly higher $(79.1 \%$ vs. $71.9 \% ; p=0.041)$. Remission rates were similar in etiologically not determined and idiopathic seizures. According to the seizure types remission rates varied with higher trends in patients with complex partial and secondary generalized tonic clonic seizures than simple partial type $(79.7 \%$ and $71.1 \%$ vs. $68.6 \%)$. The number of seizure free patients also increased from 0 at baseline to 109 (36.3\%) at month 1 and $175(58.3 \%)$ at month 3. At the end of 6 months the overall retention rate was $87.3 \%$ with slight insignificant higher trend in adults than children $(88.6 \%$ vs. $85.2 \%$ ). On brief neurological examination no abnormality was reported by the investigators at the end of the study. Clinical Global Impression (CGI) assessment data was not available for 79 cases $(26.3 \%)$ however improvement was rated as "marked" in $86.8 \%$ and "moderate" in $13.1 \%$ of cases on the basis of available data. No severe adverse event was reported in this study. However, $9.3 \%$ of patients experienced side effects like drowsiness and weight gain.

\section{Discussion}

The purpose of this study was to collect additional data on the effectiveness of valproate sustained-release as first-line monotherapy in patients with partial epilepsy in Bangladesh. The subjects were enrolled consecutively by 5 investigators at their daily neurological practice. Adults and children 6 years and above newly or recently diagnosed with partial epilepsy with or without secondary generalized seizures for whom investigators decided to prescribe valproate sustained-release as first-line monotherapy were included in this study. The primary endpoint was the remission rate defined as the proportion of seizure- free patients at 6 months. Remission rate at 1 and 3 months were also evaluated. The overall remission rate at 6 months of valproate sustained-release was $74.7 \%$ (224). In children the rate was significantly higher $(79.1 \%$ vs. $71.9 \%$; $=0.041)$. The number of seizure free patients also increased from 0 at baseline to $109(36.3 \%)$ at month 1 and $175(58.3 \%)$ at month 3 . At the end of 6 months the overall retention rate was $87.3 \%$ with small higher trend in adults $(88.6 \%)$ than children $(85.2 \%)$. Only $9.3 \%$ of patients experienced side effects like drowsiness and weight gain. No severe adverse event was reported. The result of valproate sustained-release therapy in this study is encouraging in terms of efficacy and tolerability profile.

\section{Conclusion}

This observational study supports that sodium valproate sustained formulation for first-line treatment of partial epilepsy in both adults and children is effective with acceptable tolerability in daily neurological practice condition.

\section{References}

1. Fisher RS, van Emde Boas W, Blume W, et al. Epileptic seizures and epilepsy: definitions proposed by the International League against Epilepsy (ILAE) and the International Bureau for Epilepsy (IBE). Epilepsia 2005; 46: 470-72

2. Ben-Menadhem E, Scheepers B, Stodieck S. from consensus to daily practice. Acta Neurol Scand 2003; 108 (suppl180): 5-15

3. Moran NF, Poole K, Bell G, et al. Epilepsy in the United Kingdom: seizure frequency and severity, anti-epileptic drug utilization and impact on life in 1652 people with epilepsy. Seizure 2004; 13: 425-433

4. Semah F, Picot MC, Derambure P, et al. The choice of antiepileptic drugs in newly diagnosed epilepsy: a national French survey. Epileptic Disorders 2004; 6: 255-265

5. Legros B, Boon P, Dejonghe P, et al. Opinion of Belgian neurologists on antiepileptic drugs: Belgian study on epilepsy treatment (BESET). Acta Neurologica Scandinavica 2007; 115: 97-103

6. Sillanpa a M. Carbamazepine. In: Shorvon SD, Perucca E, Fish D, Dodson E, eds. The Treatment of Epilepsy, 2nd edn. Blackwell Science Ltd., Oxford, 2004: 345-357

7. Beydoun A, Sackellares JC, Shu V. Safety and efficacy of divalproex sodium monotherapy in partial epilepsy: a double-blind, concentrationresponse design clinical trial. Depakote Monotherapy for Partial Seizures Study Group. Neurology 1997; 48: 182-188

8. Christe W, Kramer G, Vigonius U, et al. A double-blind controlled clinical trial: oxcarbazepine versus sodium valproate in adults with newly diagnosed epilepsy. Epilepsy Research 1997; 26: 451-460

9. Richens A, Davidson DL, Cartlidge NE, Easter DJ. A multicentre comparative trial of sodium valproate and carbamazepine in adult onset epilepsy. Adult EPITEG Collaborative Group. Journal of Neurology, Neurosurgery, and Psychiatry 1994; 57: 682-687

10. Verity CM, Hosking G, Easter DJ. A multicenter comparative trial of sodium valproate and carbamazepine in paediatric epilepsy. The Paediatric EPITEG Collaborative Group. Developmental Medicine and Child Neurology 1995; 37: 97-108

11. Jedrzejczak J, Kuncikova M, Magureanu S. An observational study of first-line valproate monotherapy in focal epilepsy. European Journal of Neurology 2008;15:66-72 